\title{
Investigations of fine-scale phylogeography in Tigriopus californicus reveal historical patterns of population divergence Christopher S Willett ${ }^{* 1}$ and Jason T Ladner ${ }^{1,2}$
}

Address: ${ }^{1}$ Department of Biology, University of North Carolina, Chapel Hill, Chapel Hill NC 27599-3280, USA and ${ }^{2}$ Current address: Hopkins Marine Station of Stanford University, Department of Biological Sciences, Oceanview Blvd, Pacific Grove 93950, USA

Email: Christopher S Willett* - willett4@email.unc.edu; Jason T Ladner - jtladner@stanford.edu

* Corresponding author

Published: 23 June 2009

BMC Evolutionary Biology 2009, 9:139 doi:10.1 |86//47|-2/48-9-139

This article is available from: http://www.biomedcentral.com/I47/-2/48/9//39

(c) 2009 Willett and Ladner; licensee BioMed Central Ltd.

This is an Open Access article distributed under the terms of the Creative Commons Attribution License (http://creativecommons.org/licenses/by/2.0), which permits unrestricted use, distribution, and reproduction in any medium, provided the original work is properly cited.
Received: 5 February 2009

Accepted: 23 June 2009

\begin{abstract}
Background: The intertidal copepod Tigriopus californicus is a model for studying the process of genetic divergence in allopatry and for probing the nature of genetic changes that lead to reproductive isolation. Although previous studies have revealed a pattern of remarkably high levels of genetic divergence between the populations of this species at several spatial scales, it is not clear what types of historical processes are responsible. Particularly lacking are data that can yield insights into population history from the finest scales of geographic resolution.

Results: Sequence variation in both cytochrome $b$ (CYTB, mtDNA) and the rieske iron-sulfur protein (RISP, nuclear) are examined at a fine scale within four different regions for populations of $T$. californicus. High levels of genetic divergence are seen for both genes at the broader scale, and genetic subdivision is apparent at nearly all scales in these populations for these two genes. Patterns of polymorphism and divergence in both CYTB and RISP suggest that selection may be leading to non-neutral evolution of these genes in several cases but a pervasive pattern of neither selection nor coadaptation is seen for these markers.

Conclusion: The use of sequence data at a fine-scale of resolution in this species has provided novel insights into the processes that have resulted in the accumulation of genetic divergence among populations. This divergence is likely to result from an interplay between a limited dispersal ability for this copepod and the temporal instability of copepod habitat. Both shorter-term processes such as the extinction/recolonization dynamics of copepod pools and longer-term processes such as geological uplift of coastline and sea level changes appear to have impacted the patterns of differentiation. Some patterns of sequence variation are consistent with selection acting upon the loci used in this study; however, it appears that most phylogeographic patterns are the result of history and not selection on these genes in this species.
\end{abstract}

\section{Background}

Genetically divergent populations of the intertidal copepod Tigriopus californicus have become an important model system for studying how populations diverge in allopatry and how this genetic divergence can lead to the accumulation of reproductive isolation between populations [1-7]. Important questions still remain to be answered in this species concerning the nature of the historical processes that have led to the dramatic levels of genetic divergence between populations. Studies of varia- 
tion in DNA sequences can be a powerful tool for intraspecific phylogeography and can help reveal historical patterns of population differentiation but have not yet been applied to the finest scales of resolution in this species, the scale over which gene flow is likely to be occurring $[2,3,5,8,9]$. An examination of this type of data may help reveal the nature of the interplay between gene flow, geography, geological processes, and extinction that contribute to genetic divergence in this system.

Despite the apparent ephemeral nature of their habitat (high intertidal rock pools that individually dry up on a regular basis), data from T. californicus populations show convincing evidence of long-term persistence within an outcrop and limited gene flow between rocky outcrops that results in the accumulation of substantial genetic differentiation between these populations [2]. Over the range of this copepod species from southern Alaska to central Baja California, Mexico, nucleotide sequences can show high levels of genetic divergence between regions on the scale of $100 \mathrm{~km}$ apart or less, which in mtDNAencoded genes can exceed twenty percent $[3,5,10,11]$. Studies of allozyme variation $[12,13]$ and transplant experiments [9] reveal that between rocky outcrops, within a region, there is also little gene flow. In fact, longer-term monitoring of differences in allozyme frequencies between outcrops (some as close as $500 \mathrm{~m}$ apart) reveal that differences remained stable for at least 18 years, potentially more than 100 copepod generations [2]. However, over time local population extinctions are likely, due to the ephemeral nature of copepod habitat $[14,15]$ and long distance gene flow must occur occasionally as evidenced by the recolonization of previously glaciated regions [5]. Most of this previous work primarily utilized allozyme markers at the finest scales of geographic resolution with the result that patterns of DNA sequence variation have not yet been studied at the geographic scale necessary to yield insights into the longerterm historical processes of extinction and recolonization.

The high levels of mtDNA divergence between populations of $T$. californicus reflect large numbers of differences in both synonymous and non-synonymous (or amino acid changing) sites. For example in the cytochrome b gene (CYTB, 1128 bp) between two California populations (Santa Cruz, SCN and Abalone Cove, $\mathrm{AB}$ ) there are 197 synonymous differences and 28 amino acid changes [10]. These high levels of divergence are likely to reflect both long periods of isolation between populations and high mutation rates. Willett and Burton [10] showed that mtDNA is evolving at a much more rapid rate than nuclear genes in this species, between 26 and 38-fold higher at synonymous sites. Although higher rates of mtDNA than nuclear DNA evolution are found in other taxa, especially vertebrates [16], the ratio of mtDNA to nuclear DNA rates of evolution in Tigriopus appears to be high when compared with other invertebrates and arthropods $[10,17,18]$. There is some evidence for positive selection acting upon a limited number of sites in one mtDNA gene in T. californicus, cytochrome oxidase subunit 2, COII [11], but in general the rapid accumulation of synonymous differences between these populations weakens the power of these rate-based tests of selection to detect selection on amino acid substitutions.

Despite the limited evidence to date for selection on mtDNA in T. californicus from patterns of sequence divergence there are reasons to believe that evolution of mtDNA in this species has resulted in the functional divergence of mtDNA-encoded proteins between populations. A number of experiments in T. californicus examining hybrid fitness and enzymatic rates have suggested that a portion of the genetic variation between populations leads to divergence in intergenomic coadaptation [6]. MtDNA-encoded proteins in the electron transport system are part of large, multi-subunit proteins and interact directly with many nuclear-encoded proteins so that intimate co-evolution between these proteins is likely to occur. Studies in T. californicus have shown that the interactions between mtDNA-encoded and nuclear-encoded proteins in the electron transport system have resulted in divergence in genomic coadaptation and include examples of the loss of functional coadaptation as measured by examining electron transport system enzyme and gene activities [19-22], impaired mitochondrial function [21], and lowered hybrid copepod fitness [21,23-28]. It is not clear if this coadaptation between mtDNA-encoded and nuclear-encoded proteins of the ETS has lead to consistent selection acting upon mtDNA and if this selection on mtDNA could alter phylogeographic patterns.

In this study we will characterize nucleotide sequence variation in CYTB (a mtDNA-encoded protein of complex III of the electron transport system) to study fine-scale phylogeography in T. californicus and the potential impacts of non-neutral evolution upon its diversification among these copepod populations. In addition, variation in a nuclear-encoded protein of complex III of the electron transport system, the rieske iron-sulfur protein (RISP) will be studied for use as a nuclear-encoded marker for phylogeographic inferences. A unique aspect of this study is that although there have been a number of studies in T. californicus examining fine-scale population structure (within and between adjacent outcrops) using allozymes and broader-scale studies (involving geographically distant populations) using both allozymes and mtDNA/nuclear gene sequences, there are few data available for sequence evolution and differentiation at the finer population scale. These sequence comparisons will have the added advantage of bringing a historical perspective to studies of population subdivision and molecular evolution. We will center these fine-scale studies of genetic differentiation on 
four different geographic regions (each of which has most likely evolved independently of the other regions for significant periods of time) to determine the extent to which patterns of divergence are repeated across regions.

\section{Methods \\ Population sampling}

To investigate genetic divergence in mtDNA and population history among closely spaced Tigriopus californicus populations, copepods were collected from high intertidal rock pools spaced along 3-16 km stretches of coastline centered at four rocky outcrops in California that have been used extensively in previous studies of T. californicus: San Diego (SD) on Point Loma and La Jolla Point (LJP) both in San Diego County, Abalone Cove (AB) on the Palos Verdes Peninsula in Los Angeles County, and Santa Cruz (SCN) in central California. Sampling at each of these four outcrops (and surrounding regions) was primarily done in one of two months, November 2002 and August 2004. For each sampling site, copepods were combined from several pools from within a single outcrop into a single sample and individuals were randomly selected from this sample for genetic analyses. Previous studies using transplanted individuals have indicated that pools located on the same rocky outcrop will tend to become genetically homogenized over periods as short as months [9]. Between two and ten other sites from disjunct outcrops were sampled from the vicinity of each of the four focal sites within each region (Figure 1 and Additional file 1, Table S1). In general all accessible outcrops with copepod pools immediately adjacent to the focal sites were sampled and then outcrops were sampled at increasing distances away. The sampling on Point Loma was not as extensive as for the other three regions, only two other outcrops to the south of the SD population were sampled.

Additional samples were taken from locations outside of these regions (we will call them "additional sites") to serve as potential outgroups for intra-region comparisons and to help clarify the relationships of regions to one another (Figure 1). With the combination of focal region sites and additional sites, the sampled populations in southern California (Los Angeles and south) include most of the major areas containing T. californicus for this region. Near Santa Cruz, the additional site at Pescardero (PES) was sampled for this study, a sampling that is not an exhaustive set of the T. californicus sites around central California. Several other geographically distant sites in California and British Columbia, Canada were also included in phylogenetic analyses for СУТВ.

\section{Sequencing Cytochrome $b$ and RISP}

Complete CYTB sequences were obtained from individual copepods from each of the sites (listed in Figure 1) to compare the extent of population divergence within and between sites and regions. Soon after collection, DNA from individual copepods was prepared using a simple proteinase-K cell-lysis method [23]. The complete CYTB gene was PCR amplified from these individuals using either a conserved set of primers or unique primer sets developed for the $\mathrm{SD}, \mathrm{AB}$, and $\mathrm{SCN}$ regions (see Additional file 2, Table S2) that amplified a product of between 1300 to $1800 \mathrm{bp}$ in size depending on the primer sets used. PCR products were directly sequenced using Big-Dye Terminator and run on a capillary sequencing machine. Direct sequencing of the complete CYTB (1131 bp) was performed for 10 or more individuals from most sites (see Table 1 for numbers); generally only two individuals were sequenced for CYTB from each of the additional sites.

For a nuclear gene comparison to CYTB, the RISP gene was sequenced from copepods from a subset of the above sites. This gene includes an 883 to $1113 \mathrm{bp}$ intron that provides extensive variation between individuals and sites. Primers to amplify nearly the complete coding sequence for this gene were previously developed ([10] and see Additional file 2, Table S2). PCR amplifications were done using PfuUltra Hotstart High-Fidelity DNA Polymerase from (Stratagene, Cedar Creek, TX). The resultant RISP PCR products were cloned using a zero blunt TOPO cloning kit (Invitrogen, Carlsbad, CA). For most individuals, both direct sequencing of the PCR products and sequencing of cloned sequences were done to both identify the heterozygous sites and the haplotype phases. Sequences of RISP of 1585, 1753, and 1838 bp (size differences result from variation in intron size) were obtained from Palos Verdes, La Jolla, and Santa Cruz region copepods respectively from at least 4 individuals from each site. Two haplotype sequences were constructed for each individual for RISP, in a few cases these haplotypes were identical for individuals that were homozygotes. RISP and CYTB sequences have been submitted to GenBank with the accession numbers GQ140634GQ141051 and are also included in the additional files (see Additional files 3 and 4).

\section{Sequence Analysis and Population Structure}

Sequences were edited and aligned using Sequencher version 4.7 (Genecodes, Ann Arbor, MI). The program DNAsp version 4.1 [29] was used to examine levels of polymorphism, divergence, and compute statistical metrics of selection (e.g. Tajima's D [30], Fu and Li's D and F [31]), compute $\mathrm{R}$, the recombination parameter [32], and $\mathrm{R}_{\mathrm{m}}$, the minimum number of recombination events [33] for the CYTB and RISP sequences. Fay and Wu's H [34] was calculated for each site using the closest available outgroup (PES for Santa Cruz, Dume for Palos Verdes, and LagBch for Point Loma and La Jolla). The significance of 


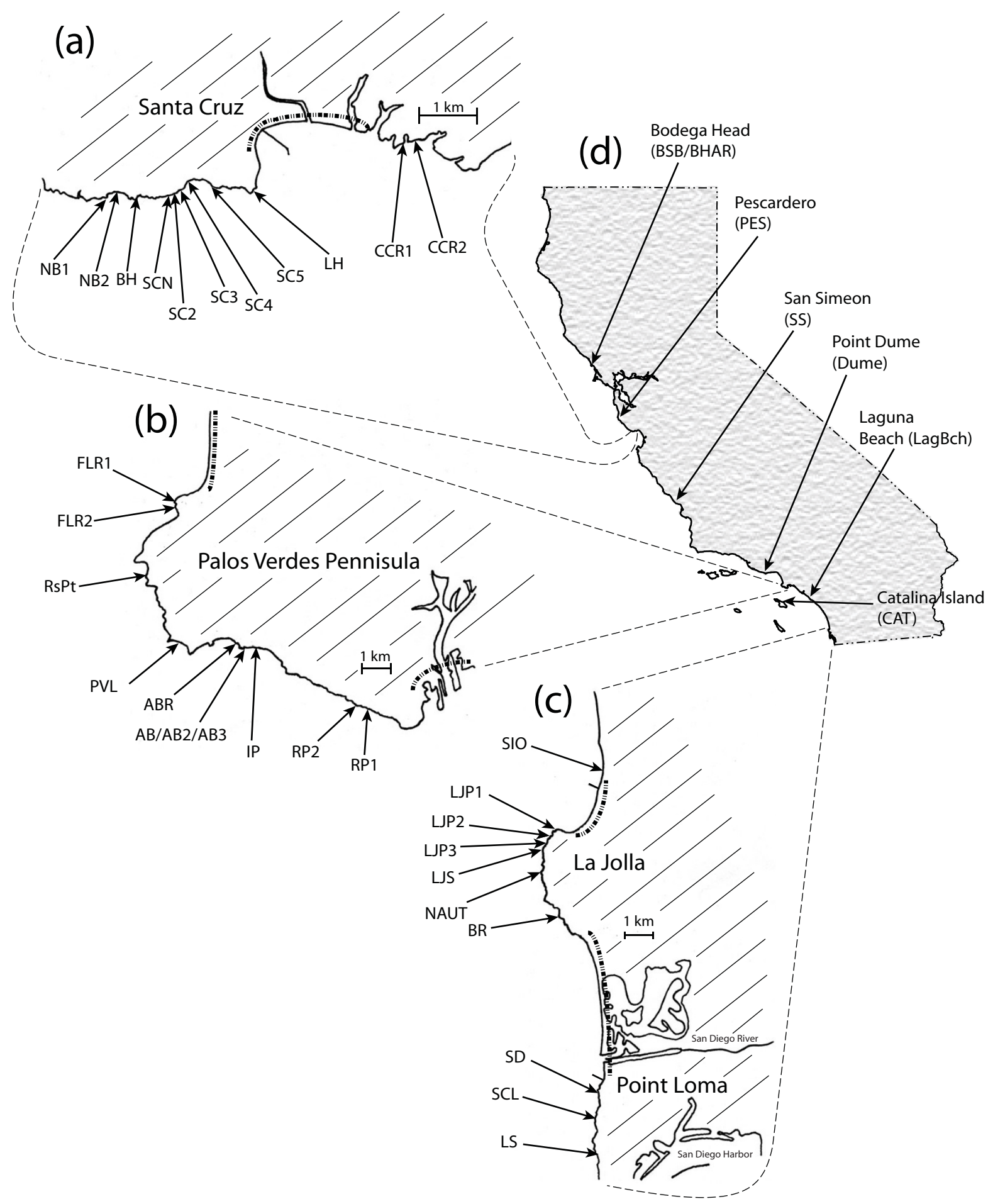

Figure I

Map of T. californicus sites along the coast in California. Sites sampled within the (a) Santa Cruz, (b) Palos Verdes, and (c) La Jolla and Point Loma regions are depicted. Dashed lines within each region indicate extensive portions of coastline that consist of low-lying stretches of sandy beach, which are unlikely to have had any copepod habitat in recent time. The location of each of these regions within California is depicted in (d) with the locations of the additional sites. Two other additional sites were included in this study from British Columbia, Canada but are not depicted on this map: Point Atkinson, West Vancouver (BC) and Ucluelet, Vancouver Island (VI). 
Table I: Polymorphism in CYTB within focal regions for T. californicus

\begin{tabular}{|c|c|c|c|c|c|c|c|c|c|}
\hline Region & Site & Indivs. & Hap. \# & Syn. ${ }^{\prime}$ & Nonsyn. & $\pi_{\text {syn }}{ }^{2}$ & $\pi_{\text {total }}$ & Taj-D ${ }^{3}$ & $\mathrm{FW}-\mathrm{H}^{4}$ \\
\hline \multirow[t]{13}{*}{ Santa Cruz } & NBI & 10 & 1 & 0 & 0 & 0 & 0 & NA & NA \\
\hline & NB2 & 10 & 3 & 1 & 1 & 0.00127 & 0.00049 & -0.69 & 0.44 \\
\hline & $\mathrm{BH}$ & 10 & 2 & 1 & 0 & 0.00127 & 0.00031 & 0.01 & -1.07 \\
\hline & SCN & 15 & 3 & 5 & 3 & $0.0044 I$ & 0.00184 & -0.57 & $-1.4 \mid$ \\
\hline & $\mathrm{SC} 2$ & 5 & 1 & 0 & 0 & 0 & 0 & $\mathrm{~N} / \mathrm{A}$ & $N / A$ \\
\hline & $\mathrm{SC} 3$ & 10 & 3 & 5 & 1 & 0.00618 & 0.00185 & -0.06 & -0.71 \\
\hline & SC4 & 10 & 2 & 3 & 3 & 0.00214 & 0.00106 & $-1.79 *$ & $-6.04 * *$ \\
\hline & SC5 & 10 & 2 & 4 & 1 & 0.00285 & 0.00088 & $-1.74 *$ & $-4.44 * *$ \\
\hline & $\mathrm{LH}$ & 10 & 2 & 1 & 2 & 0.00198 & 0.00147 & $2.06 *$ & 0 \\
\hline & CCRI & 10 & 3 & 6 & 2 & 0.00428 & 0.00141 & $-1.88^{*}$ & -2.13 \\
\hline & CCR2 & 10 & 3 & 6 & 3 & 0.00487 & 0.00173 & $-1.68^{+}$ & -1.42 \\
\hline & Total & 110 & 12 & 10 & 6 & 0.00996 & 0.00359 & 1.17 & 1.11 \\
\hline & Avg. & & 2.27 & & & 0.00266 & 0.0010 & & \\
\hline \multirow[t]{13}{*}{ Palos Verdes } & FRI & 10 & 4 & 3 & 1 & 0.00308 & 0.00094 & -0.9 & $-5.42 * *$ \\
\hline & FR2 & 10 & 3 & 14 & 1 & 0.02316 & 0.00601 & 1.3 & -1.07 \\
\hline & $\mathrm{RsPt}$ & 10 & 7 & 9 & 0 & 0.00929 & 0.00230 & 0.8 & $-4.89 *$ \\
\hline & PVL & 10 & 3 & 1 & 2 & 0.0007 I & 0.00083 & -0.43 & -1.6 \\
\hline & $A B R$ & 10 & 1 & 0 & 0 & 0 & 0 & N/A & $N / A$ \\
\hline & $A B I$ & 11 & 5 & 8 & 1 & 0.01003 & 0.00287 & 0.22 & 1.31 \\
\hline & $A B 2$ & 11 & 7 & 10 & 2 & 0.00861 & 0.00281 & -0.98 & -0.44 \\
\hline & $A B 3$ & 10 & 8 & 12 & 3 & 0.00859 & 0.00267 & $-2^{* *}$ & $-3.2^{*}$ \\
\hline & IP & 10 & 5 & 7 & 1 & 0.00559 & 0.00156 & -1.64 & $-4^{*}$ \\
\hline & RPI & 10 & 6 & 9 & 2 & 0.00996 & 0.00309 & -0.46 & $-2.76^{+}$ \\
\hline & RP2 & 10 & 5 & 2 & 3 & 0.00272 & 0.00135 & 0.02 & 0.71 \\
\hline & Total & 112 & 47 & 73 & 14 & 0.03399 & 0.00912 & -1.08 & $-40.3 * * *$ \\
\hline & Avg. & & 4.91 & & & 0.00743 & 0.00222 & & \\
\hline \multirow{9}{*}{ La Jolla } & $\mathrm{SIO}$ & 14 & 3 & 1 & 1 & 0.00051 & 0.00036 & -0.96 & 0.35 \\
\hline & LJPI & 20 & 10 & 9 & 4 & 0.00753 & 0.00265 & -0.66 & $-2.85^{+}$ \\
\hline & LJP2 & 13 & 5 & 11 & 1 & 0.00973 & 0.00263 & -0.94 & $-7.02 *$ \\
\hline & LJP3 & 10 & I & 0 & 0 & 0 & 0 & $\mathrm{~N} / \mathrm{A}$ & N/A \\
\hline & LJS & 14 & 6 & 5 & 1 & 0.00299 & 0.00086 & -1.73 & -1.09 \\
\hline & NAUT & 13 & 4 & 10 & 0 & 0.00556 & 0.00136 & $-2.09 * *$ & $-2.39 *$ \\
\hline & $B R$ & 14 & 7 & II & 1 & 0.01426 & 0.00361 & 0.33 & $-3.47^{+}$ \\
\hline & Total & 98 & 32 & 37 & 7 & 0.0173 & 0.00457 & -0.87 & $-8.9 *$ \\
\hline & Avg. & & 5.14 & & & 0.00580 & 0.00164 & & \\
\hline \multirow[t]{5}{*}{ Point Loma } & $S D$ & 20 & 7 & 13 & 0 & 0.00793 & 0.00193 & -1.46 & -1.21 \\
\hline & $\mathrm{SCL}$ & 7 & 3 & 2 & I & 0.00206 & 0.00076 & -1.35 & -0.95 \\
\hline & LS & 8 & 2 & 1 & 0 & 0.00090 & 0.00022 & -1.05 & 0.21 \\
\hline & Total & 35 & 12 & 16 & 1 & 0.00787 & 0.00198 & -1.39 & -0.88 \\
\hline & Avg. & & 4.00 & & & 0.00363 & 0.00097 & & \\
\hline
\end{tabular}

INumber of synonymous polymorphisms (syn.) or non-synonymous (nonsyn.)

${ }^{2}$ Average number of pairwise differences between sequences within a population for synonymous $\left(\pi_{\text {syn }}\right)$, non-synonymous $\left(\pi_{\text {non-syn }}\right)$, and all $\left(\pi_{\text {total }}\right)$ sites.

3Tajima's $\mathrm{D}$ value with significance indicated by an $*(\mathrm{P}<0.05)$ or an $* *(\mathrm{P}<0.0 \mathrm{I})$, and + indicating $0.05<\mathrm{P}<0 . \mathrm{I}$.

${ }^{4}$ Fay and Wu's $\mathrm{H}$ value with significance indicated by an $*(\mathrm{P}<0.05)$, an $* *(\mathrm{P}<0.0 \mathrm{I})$, or an $* * *(\mathrm{P}<0.00 \mathrm{I})$, and + indicating $0.05<\mathrm{P}<0 . \mathrm{I}$.

H was tested by running coalescent simulations in DNAsp using up to 10000 replicates with no recombination for CYTB and several different parameter values of moderate recombination for RISP. McDonald and Kreitman tests (MK test [35]) were performed using divergence and polymorphism data from DNAsp and hand counts. The program Arlequin v3.11 [36] was used to compute analyses of molecular variance (AMOVA) to partition variation between sites, regions, and individuals, and to calculate pairwise $\mathrm{F}_{\mathrm{ST}}$ values between sites based on genetic diver- gence and their significance (using 10000 permutation replicates) for CYTB and RISP. Isolation by distance testing was performed using the IBD web service version 3.02 with the significance assessed using a Mantel test [37]. Nested clade analysis was implemented using the program GeoDis version 2.2 [38] for the CYTB dataset using the inference key of Templeton [39]. Multilocus nested clade analysis was not attempted for the combined RISP/ $C Y T B$ datasets due to extensive recombination within regions for RISP. 
Phylogenetic analyses were conducted using several different methods: Haplotype networks were constructed for CYTB by hand using a parsimony method and also using statistical parsimony via the program TCS v1.18 [40]. Gene trees for the CYTB sequences were constructed under the parsimony criterion using the program PAUP* version $4.0 \mathrm{~b} 10$ [41]. A Bayesian tree was also constructed for the same set of CYTB sequences using the program MrBayes v3.1.2 [42]. For this analysis, a GTR model with gammadistributed rate variation and a proportion of invariant sites was run for 800000 generations with a 200000 generation burn-in time (sampled every 1000 generations). Alternate models from GTR were not explored with Bayesian analyses. Gene trees for RISP were done with PAUP* using a neighbor-joining analysis (NJ). A full parsimony analysis of all sequences could not be completed for RISP in a single analysis most likely due to a history of recombination between alleles within regions. Analyses for each region with subsets of sequences from all other major clades could be completed in separate analyses using parsimony and the correspondence between the relationships found in these parsimony analyses and those found in the $\mathrm{NJ}$ tree was then determined.

\section{Results \\ CYTB variation within and between regions}

Phylogenetic analyses of CYTB sequences reveal deep splits among the four different focal regions, with considerably less variation occurring among sites within each region (Figure 2). Pairwise divergences among regions are between 19 and 22 percent (see Additional file 5, Table S3; with the exception of individuals from the Point Loma and La Jolla sites that have $10.2 \%$ average divergence and are only $8 \mathrm{~km}$ apart). Although the Palos Verdes region is the closest geographically to each of the CAT, Dume, and LagBch sites, it is highly diverged from each at CYTB. In contrast, comparisons of select other geographically distant sites show much lower genetic divergence-LagBch and Point Loma sites (2.3\% divergence, $107 \mathrm{~km}$ apart), PES and Santa Cruz sites (1.0\% divergence, $47 \mathrm{~km}$ apart), and Dume and Cat sites (3.1\% divergence, $68 \mathrm{~km}$ apart). Within each of the four focal regions, pairwise divergences among individuals from different sites are much lower (below 1.3\%), and levels of polymorphism within individual sites are relatively low as well (below 0.7\%) (Table 1). An analysis of molecular variation (AMOVA) shows that although most variation at CYTB is partitioned between regions, there is also significant variation between sites within regions as well (Table 2a).

There is significant genetic structure in each region evident at scales down to the site to site comparisons but also some difference in the degree of haplotype sharing across each region. The Santa Cruz region shows the greatest degree of CYTB haplotype sharing across sites with three common haplotypes (Figure 3 ) that are shared by at least three different sites. Individuals from geographically separated sites share haplotypes (e.g. CCR1/2 and SCN share haplotype I, and NB1/2 and LH share haplotype III), which results in no significant correlation between genetic distance (FST) and geographic distance within this region $(\mathrm{r}=0.092, \mathrm{p}=0.25)$ despite general pairwise FST values among sites higher than 0.5 (all CYTB FST values in Additional file 6, Table S4). However, there are two groups of Santa Cruz sites that do not show significant pairwise FST values for comparisons of sites within each group (significance assessed by permutation tests and significance threshold adjusted using a sequential Bonferroni procedure to adjust for multiple tests). One group is the SCN/ SC3/CCR1 sites that share haplotype I (SC3 is also not significantly differentiated from the SC2 and SC5 sites), while a second group, the NB1/NB2/SCN4/BH/LH sites, share haplotype III.

The two San Diego county regions show different patterns in their CYTB haplotypes than the Santa Cruz region. The

Table 2: Differences between regions, sites, and individuals for CYTB and RISP in an AMOVA

\begin{tabular}{|c|c|c|c|c|c|c|c|}
\hline Comparison ${ }^{1}$ & Source & d.f. & SS & Variance Components & $\%$ variation & Fixation index & P-value \\
\hline \multirow[t]{3}{*}{ a. CYTB-among regions } & Among regions & 3 & 27574 & 107.70 & 96.8 & $\mathrm{~F}_{\mathrm{CT}}=0.968$ & $<0.0001$ \\
\hline & Among sites within regions & 28 & 823 & 2.61 & 2.34 & $\mathrm{~F}_{\mathrm{ST}}=0.991$ & $<0.0001$ \\
\hline & Within sites & 323 & 312 & 0.97 & 0.87 & & $<0.0001$ \\
\hline \multirow[t]{3}{*}{ b. CYTB-within Palos Verdes } & Among non-adjacent groups & 2 & 247.1 & 4.65 & 71.19 & $\mathrm{~F}_{\mathrm{CT}}=0.712$ & 0.0023 \\
\hline & $\begin{array}{l}\text { Among sites within non-adjacent } \\
\text { groups }\end{array}$ & 5 & 28.3 & 0.41 & 6.24 & $\mathrm{~F}_{\mathrm{ST}}=0.774$ & $<0.0001$ \\
\hline & Within sites & 74 & 109 & 1.47 & 22.57 & & $<0.0001$ \\
\hline \multirow[t]{3}{*}{ c. RISP-among regions } & Among regions & $2^{2}$ & 6595 & 143.8 & 96.5 & $\mathrm{~F}_{\mathrm{CT}}=0.965$ & 0.009 \\
\hline & Among sites within regions & 6 & 91.7 & 1.44 & 0.97 & $\mathrm{~F}_{\mathrm{ST}}=0.975$ & $<0.0001$ \\
\hline & Within sites & 63 & 234.4 & 3.72 & 2.50 & & $<0.0001$ \\
\hline
\end{tabular}

'For both (a) and (c) the levels tested are the regions, sites, and individuals. In (b) the Palos Verdes region is considered because for this region it is possible to define subregional grouping based on habitat and sampling schemes [adjacent groups were defined as follows: (AB, AB2, $A B 3, A B R)$, (RPI, RP2), and (FRI, FR2). AMOVA were conducted in Arlequin using pairwise distances between populations.

2The Point Loma region contained only the SD site for the RISP results and was therefore not included as a separate group. 


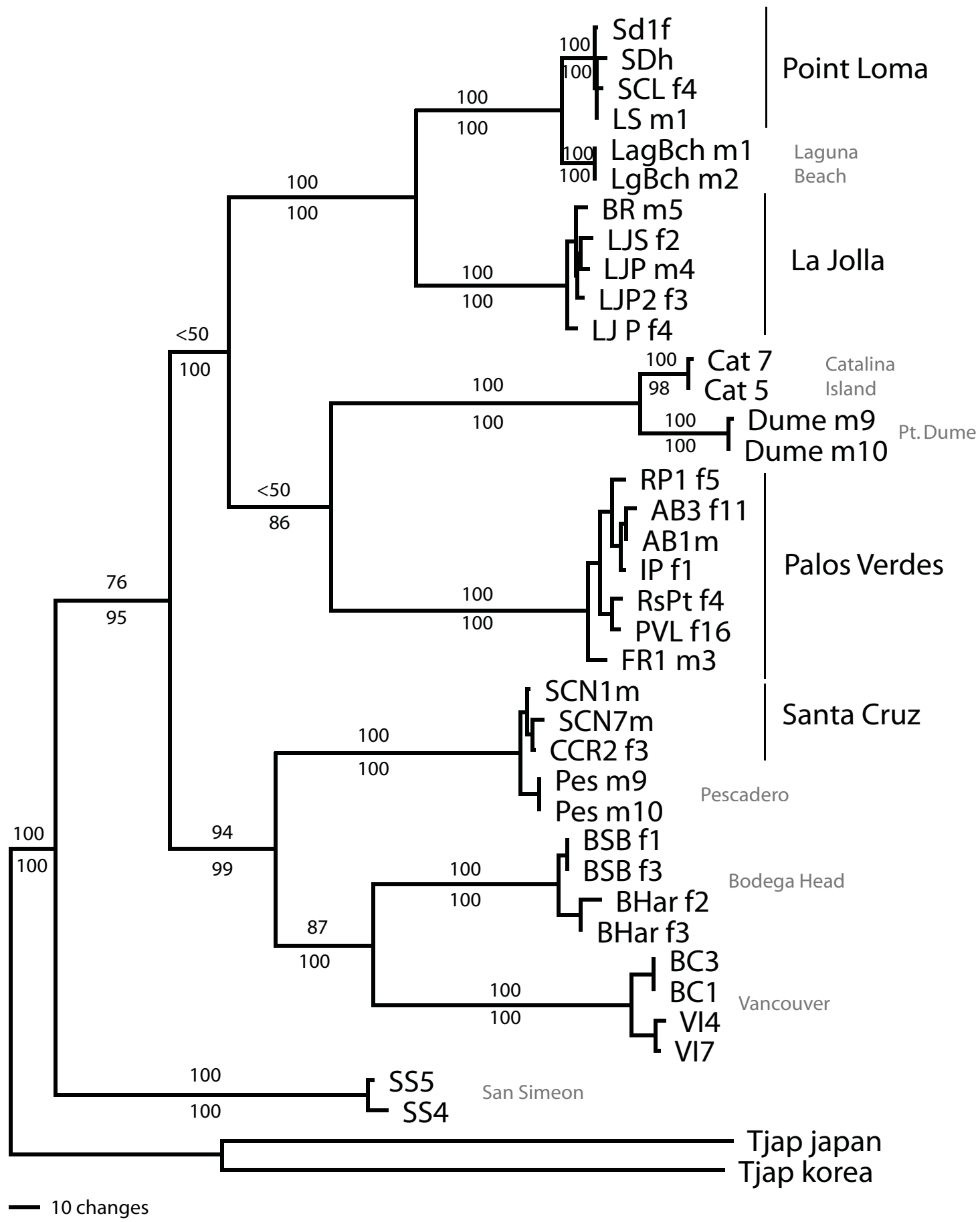

Figure 2

Gene tree for CYTB for selected sites of T. californicus. Tree is one of 16 most parsimonious trees based on the complete nucleotide sequence of CYTB (II3I bP) for taxa representing the range of diversity found within each of the regional areas and the additional site sequences. Bootstrap numbers based upon 10000 replicates are given above branches (only those supporting regional clades and branches showing the relationships of additional sites are shown). T. japonicus nucleotide CYTB sequences from both Korea and Japan are used to root tree (from GenBank accession numbers $\underline{A}$ Y 959338 and $\underline{A B 060648}$ respectively). The relationships between the regions and additional sites are the same as those obtained in a Bayesian phylogenetic analysis. Credibility values from this analysis are given below each branch. 
Figure 3

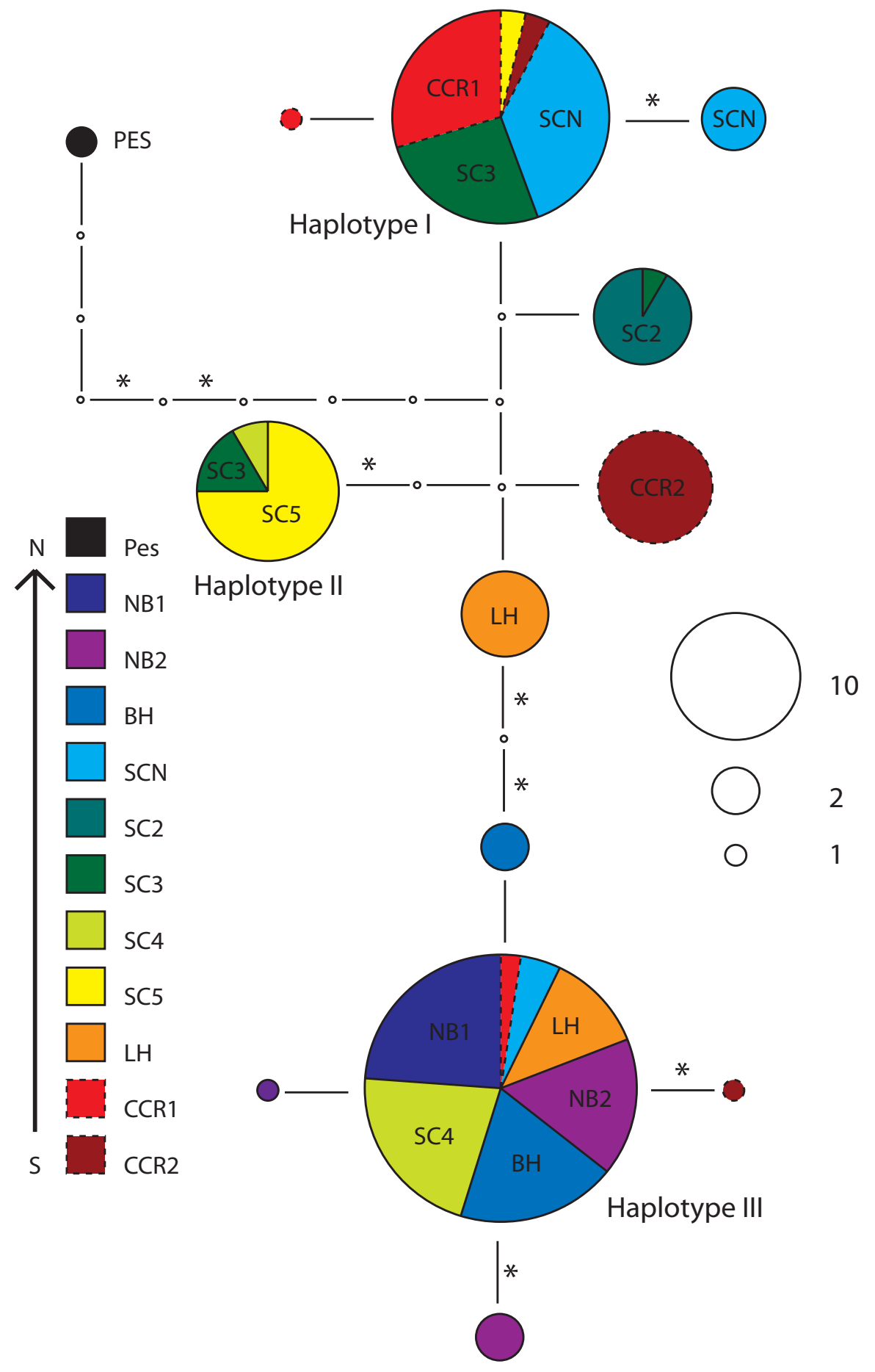

Figure 3

CYTB haplotype network for Santa Cruz region T. californicus. Each segment represents one nucleotide difference with non-synonymous changes indicated by an *. Size of circle indicates the number of haplotypes while the colors depict the sites from which these haplotypes come. Sites are shown on the network when more than two individuals from one site have the same haplotype. The haplotypes labeled I, II, and III are shared by three or more sites. Dashed lines surrounding some haplotypes are solely for visual clarity. 
La Jolla region displays an intermediate level of haplotype sharing (Figure 4a) with one common haplotype (haplotype IV) shared by five different sites, but clear geographic clustering for three other groups of closely related haplotypes. In contrast to the Santa Cruz haplotypes where several non-synonymous differences are located on internal branches, in the La Jolla region non-synonymous differences are largely found at or near tips and not on the branches separating the four main groups of haplotypes. Although there is less haplotype sharing within this region than the Santa Cruz region, there is no signature of isolation by distance $(r=-0.045, p=0.62)$. Only one group of sites showed non-significant differentiation in pairwise comparisons of $\mathrm{F}_{\mathrm{ST}}$, the LJS/NAUT/LJP3/SIO sites, all of which have haplotype IV at high frequency. $\mathrm{F}_{\mathrm{ST}}$ values are much higher $(>0.5)$ for comparisons among all other sites within La Jolla. Although not sampled as extensively, the nearby Point Loma region does not show any shared haplotypes among its three sites, but there is also no discrete clustering of haplotypes by site either (Figure $4 \mathrm{~b}$ ). The $\mathrm{F}_{\mathrm{ST}}$ values between these three sites on Point Loma range from 0.49-0.63.

The Palos Verdes region shows the greatest intra-region divergences for CYTB haplotypes with little haplotype sharing beyond adjacent sites (Figure 5). There are four clusters of haplotypes, which each contain adjacent sites and a fifth cluster of haplotypes that contains the geographically separated PVL/RsPt/FR2 sites (the FR2 site has individuals in two different haplotype clusters). Two of the 24 substitutions separating these five divergent haplotype clusters are non-synonymous. AMOVA for the Palos Verdes region (Table $2 \mathrm{~b}$ ) shows that a large fraction of the genetic variation was between non-adjacent groups and contributes to a strong signal of isolation by distance $(\mathrm{r}=$ $0.57 \mathrm{p}<0.0001$ ). Two groups of adjacent sites do not show significant differentiation by $\mathrm{F}_{\mathrm{ST}}$ measures, the $\mathrm{AB} /$ $\mathrm{AB} 2 / \mathrm{AB} 3$, and RP1/RP2 sites. All other $\mathrm{F}_{\mathrm{ST}}$ values for pairwise comparisons among sites were higher than 0.3 .

There is no evidence for major short-term temporal shifts in haplotypes within sites from the replicate sampling of individual sites at different time points. CYTB sequences obtained from copepods collected two years apart from the SCN, AB2, and LJP sites were not appreciably different from one another within a site (results not shown). This suggestion of short-term stability mirrors the longer-term stability in haplotype frequency (18 years or more) found for many of these same outcrops using allozyme markers [12].

\section{Nested clade analyses for CYTB}

Nested clade analyses using CYTB within each of the four studied regions show patterns of differentiation that are consistent with inferences of restricted gene flow and fragmentation (Table 3). A number of clade levels appear to show the signature of past long distance colonization, fragmentation, or past contiguous range expansion (seven total) indicating the importance of historical events on current population structure. For an equal number of cases (seven), the patterns of variation are consistent with either historical separation or lowered levels of gene flow (e.g., isolation by distance or restricted gene flow).

\section{Tests for Selection acting upon CYTB}

MK tests [35] reveal a pattern of variation consistent with excess non-synonymous polymorphism for 3 of the 4 regions (Table 4). For variation in CYTB in T. californicus Tajima's D values are biased towards negative values, 22 negative (5 significant) and 6 positive (1 significant) (Table 1). Fu and Li's F and D have a similar pattern of skew towards negative values (see Additional file 7, Table S5). If the frequency spectra of replacement sites alone are examined, Tajima's D values become slightly more negative for the Palos Verdes and La Jolla regions as a whole (the same pattern was not found overall across individuals sites; see Additional file 7, Table S5). Fay and Wu's H values [34] are significantly negative for the same five sites as Tajima's D and Fay and Wu's $\mathrm{H}$ is also significant at the RsPt, IP, and FR1 sites (Table 1).

\section{Variation for RISP within and among Regions}

We have sequenced RISP from copepods from a subset of the regions and sites that capture much of the diversity of haplotypes found for CYTB. A NJ tree constructed from the RISP sequences illustrates the general patterns of divergence (Figure 6). There is substantial divergence among regions, but absolute levels of sequence divergence between regions for RISP are not nearly as high as those seen for CYTB. For example, for synonymous site comparisons between the Palos Verdes region and Santa Cruz region uncorrected pairwise divergence is 70 percent for CYTB and 5.5 percent for RISP. For the RISP gene tree (Figure 6) within each region there is much less clustering of haplotypes for single sites or groups of sites than was seen for CYTB (Figure 2).

With the exception of the Santa Cruz region there is very little haplotype sharing either within a site or between sites for RISP, but individual polymorphisms are often shared across sites within a region. Compared to CYTB, for RISP there is somewhat less genetic divergence between sites within a region as reflected by the lower percentage of variation among sites within a region in an AMOVA for RISP (Table 2c) and the limited geographic structure within regions evident in the NJ tree (Figure 6). For the two sites in the Santa Cruz region there are only 7 singleton polymorphisms segregating at RISP (and the two sites are not significantly genetically differentiated at RISP; all RISP pairwise $\mathrm{F}_{\mathrm{ST}}$ values are in Additional file 8, Table S6). In contrast, for the 4 alleles obtained for the additional site to the north, PES, there is much more var- 
(a) La Jolla
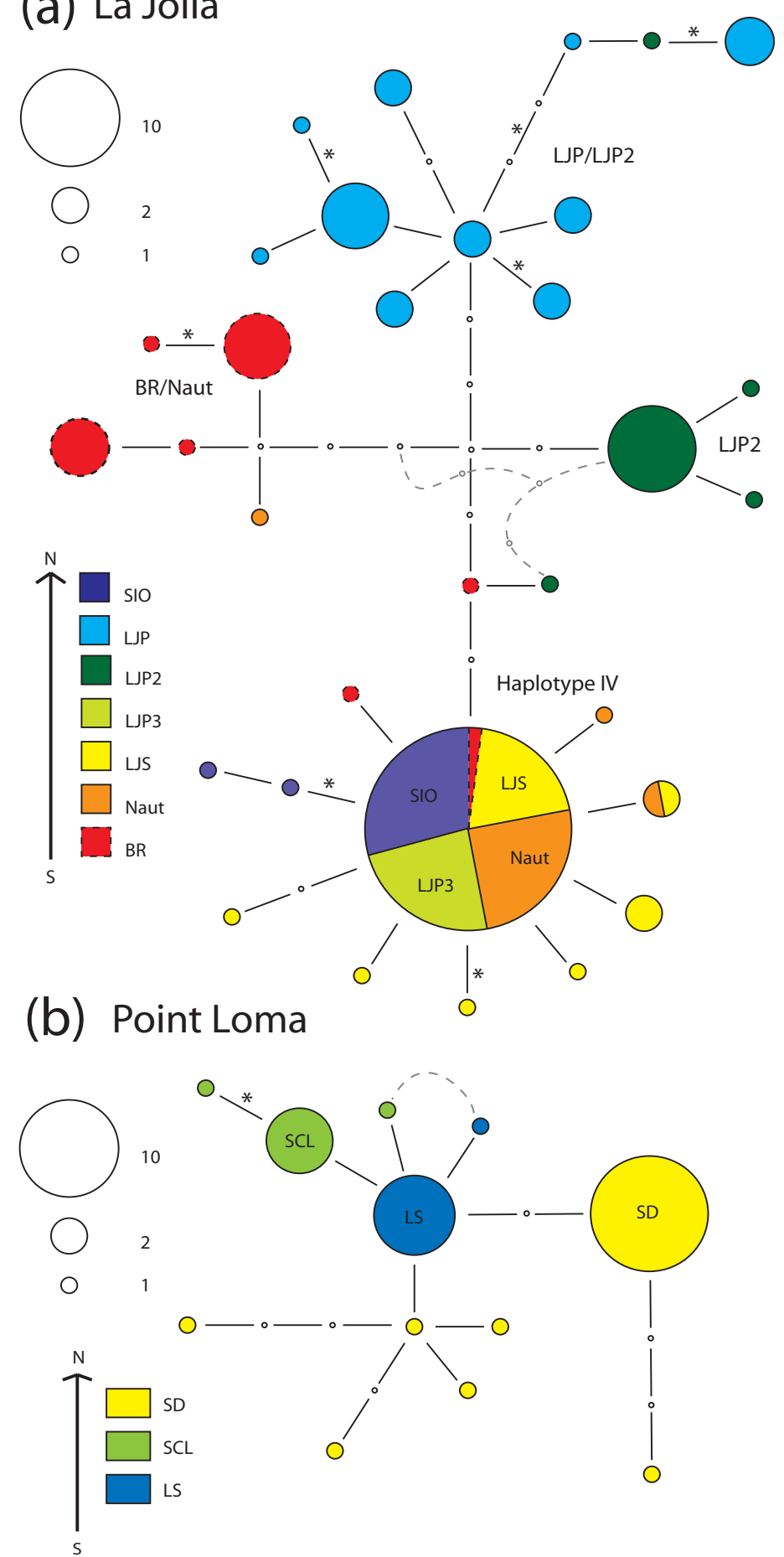

\section{Figure 4}

CYTB haplotype network for La Jolla (a) and Point Loma (b) regions for T. californicus. Network is depicted as described in Figure 3. For La Jolla, the three groups of haplotypes that cluster by geography are labeled in addition to the haplotype IV that is shared by five sites. For Point Loma, haplotypes are labeled by site when more than two individuals share a haplotype from the same site. There are 23 synonymous and I non-synonymous substitutions in CYTB separating Point Loma and Laguna Beach (and IOI synonymous and 6 non-synonymous substitutions separating Point Loma and La Jolla). Dashed lines indicate alternative haplotype relationships found in statistical parsimony analyses. 
Figure 5

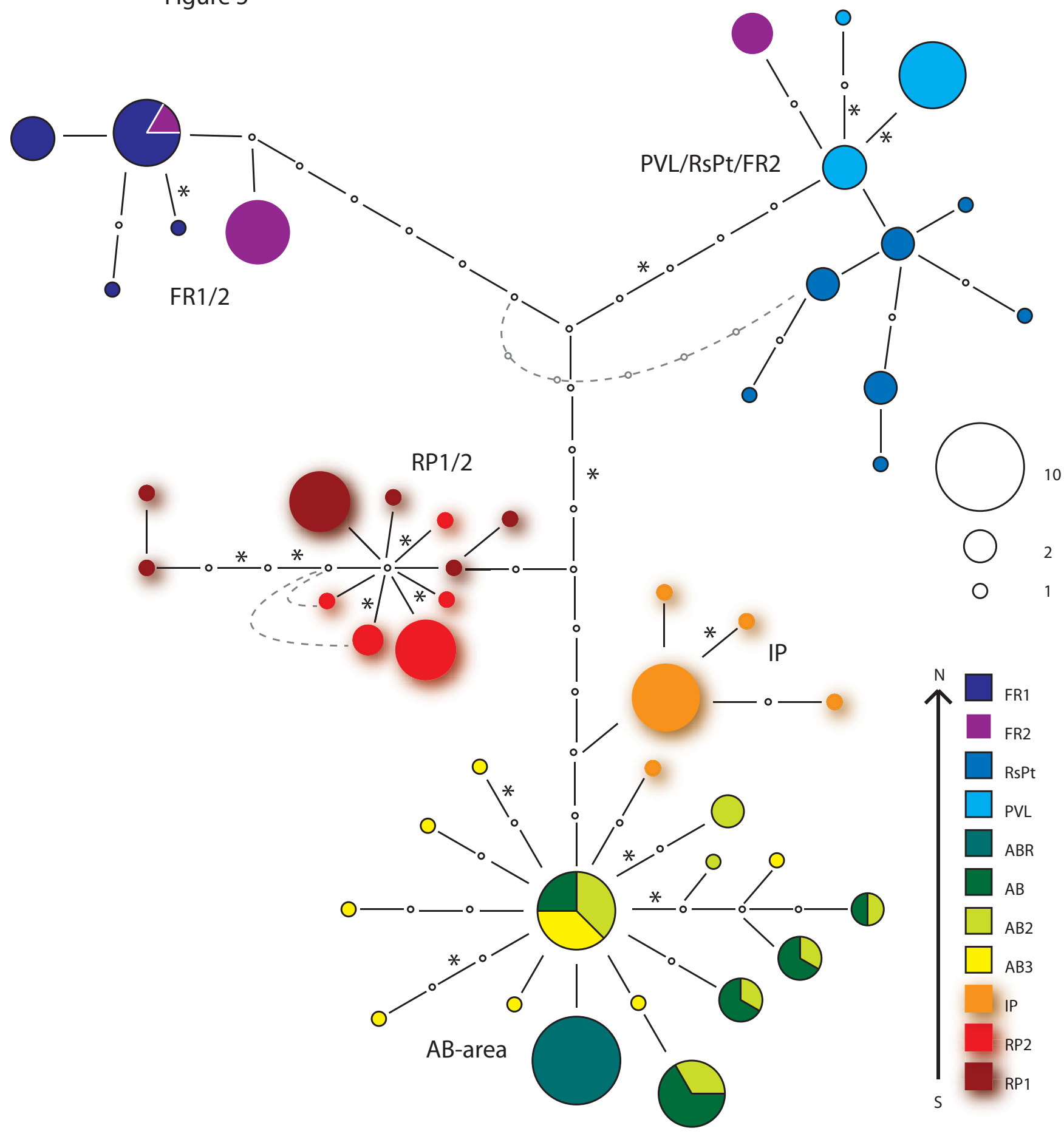

Figure 5

CYTB haplotype network for Palos Verdes region T. californicus. Network is constructed as depicted in Figure 3. Five clusters of haplotypes that group largely by geography are labeled by sites. Dashed lines indicate alternative haplotype relationships found in statistical parsimony analyses. Haplotypes are shadowed for visual clarity. 
Table 3: Nested clade analyses inferences for T. californicus sites based on CYTB.

\begin{tabular}{|c|c|}
\hline Inference & Description of clades involved \\
\hline \multicolumn{2}{|l|}{ Santa Cruz and PES } \\
\hline Inconclusive & Haplotype I, CCRI, and SCN \\
\hline Fragmentation & $\mathrm{SC} 3 / 4 / 5$ and CCR2 \\
\hline Restricted gene flow or isolation by distance & $\mathrm{CCRI} / 2$ and SCN/3/5 \\
\hline Restricted gene flow or isolation by distance & Haplotype II group and SC3/4/5/CCR2 \\
\hline Inconclusive & PES and Haplotype I group \\
\hline \multicolumn{2}{|l|}{ Palos Verdes } \\
\hline Contiguous range expansion & $A B$ to $A B R$ \\
\hline Contiguous range expansion or restricted gene flow & $\mathrm{RPI}$ and RP2 \\
\hline Contiguous range expansion or restricted gene flow & $A B / A B 2 / A B 3$ and $A B 2 / A B 3$ \\
\hline Long distance colonization & FR2 and PVL \\
\hline Contiguous range expansion & $\mathrm{FR} 1 / \mathrm{FR} 2$ and FR2 \\
\hline Fragmentation or isolation by distance & $\mathrm{PVL} / \mathrm{FR} 2$ and $\mathrm{RsPt}$ \\
\hline Long distance colonization & $\mathrm{AB} / \mathrm{IP}$ and IP groups \\
\hline Fragmentation or isolation by distance & $\mathrm{RP}, \mathrm{FR}$, and PVL/RsPt groups \\
\hline \multicolumn{2}{|l|}{ La Jolla } \\
\hline Long distance colonization or range expansion & BR group and haplotype III group \\
\hline Contiguous range expansion or restricted gene flow & Among LJP \\
\hline \multicolumn{2}{|l|}{ Point Loma and LgBch } \\
\hline Contiguous range expansion & SCL and LS \\
\hline Long distance colonization & SD and SD/SCL/LS \\
\hline
\end{tabular}

The historical and demographic inferences are listed with a general description of the clade/nesting groups involved.

iation, including an allele (m9-B) that appears to be a past recombinant between PES and Santa Cruz allele-types (see Additional file 9, Table S7). In the La Jolla region, 19 of 22 non-singleton polymorphisms are shared across sites (see Additional file 10, Table S8). The $\mathrm{F}_{\mathrm{ST}}$ values indicate significant but relatively low levels of differentiation between these three La Jolla sites (with LJS and LJP having the lowest $\mathrm{F}_{\mathrm{ST}}$ value: 0.064 despite having an $\mathrm{F}_{\mathrm{ST}}$ of 0.74 for CYTB). The Palos Verdes region sites show the least shared polymorphism with only 9 of 21 non-singletons shared across sites (see Additional file 11, Table S9). Compared to the other two regions, pairwise differentiation is highest among Palos Verdes sites as measured by $\mathrm{F}_{\mathrm{ST}}$ values, which range from 0.28 and 0.36 for IP/RP1 and IP/ PVL to 0.75 for RP1/AB.

\section{Patterns of Polymorphism in RISP}

All of the polymorphisms within regions for RISP are either in non-coding positions (primarily the large intron) or in synonymous sites in the coding regions (Table 5). As expected from the lack of haplotype variation, the Santa Cruz sites show the lowest levels of nucleotide polymorphism with the average $\pi_{\text {tot-sil }} 10$-fold lower than the corresponding value from the La Jolla sites. Estimates of intragenic recombination from both the recombination parameter $(\mathrm{R})$ and minimum number of recombination events $\left(R_{m}\right)$ are both non-zero in the Palos Verdes and La Jolla samples indicating a history of recombination in these samples of alleles within regions. The lack of variation within the Santa Cruz region precludes an estimation of recombination rate within that region.

The frequency-spectra of polymorphisms for RISP in these different sites and regions of $T$. californicus are not as biased towards low frequency polymorphisms as seen for CYTB (Table 5). For Tajima's D there are 6 positive values and 3 negative with no significant values. The only significant values for Fu and Li's D and F are from the PVL site and are positive (1.56 D and $1.64 \mathrm{~F}, \mathrm{p}<0.05$ for each; see Additional file 7, Table S5). Significantly negative values

Table 4: McDonald/Kreitman tests of polymorphism versus divergence for CYTB.

\begin{tabular}{|c|c|c|c|c|c|c|}
\hline Region & Outgroup & Fixed syn. & Fixed non-syn. & Poly. syn. & Poly. Non-syn. & P-value \\
\hline Santa Cruz & BSB & 148 & 17 & 10 & 6 & $<0.005$ \\
\hline Santa Cruz & BSB' & 260 & 17 & 10 & 6 & $<0.005$ \\
\hline Santa Cruz & PES & 7 & 2 & 10 & 6 & $>0.5$ \\
\hline Palos Verdes & Dume & 166 & 30 & 73 & 14 & 0.14 \\
\hline Palos Verdes & Dumel & 336 & 31 & 73 & 14 & 0.03 \\
\hline La Jolla & LgBch & 92 & 5 & 37 & 7 & 0.04 \\
\hline Point Loma & LgBch & 23 & I & 16 & I & $>0.5$ \\
\hline
\end{tabular}

'A Jukes/Cantor correction for multiple hits has been applied to the divergence results for these rows 


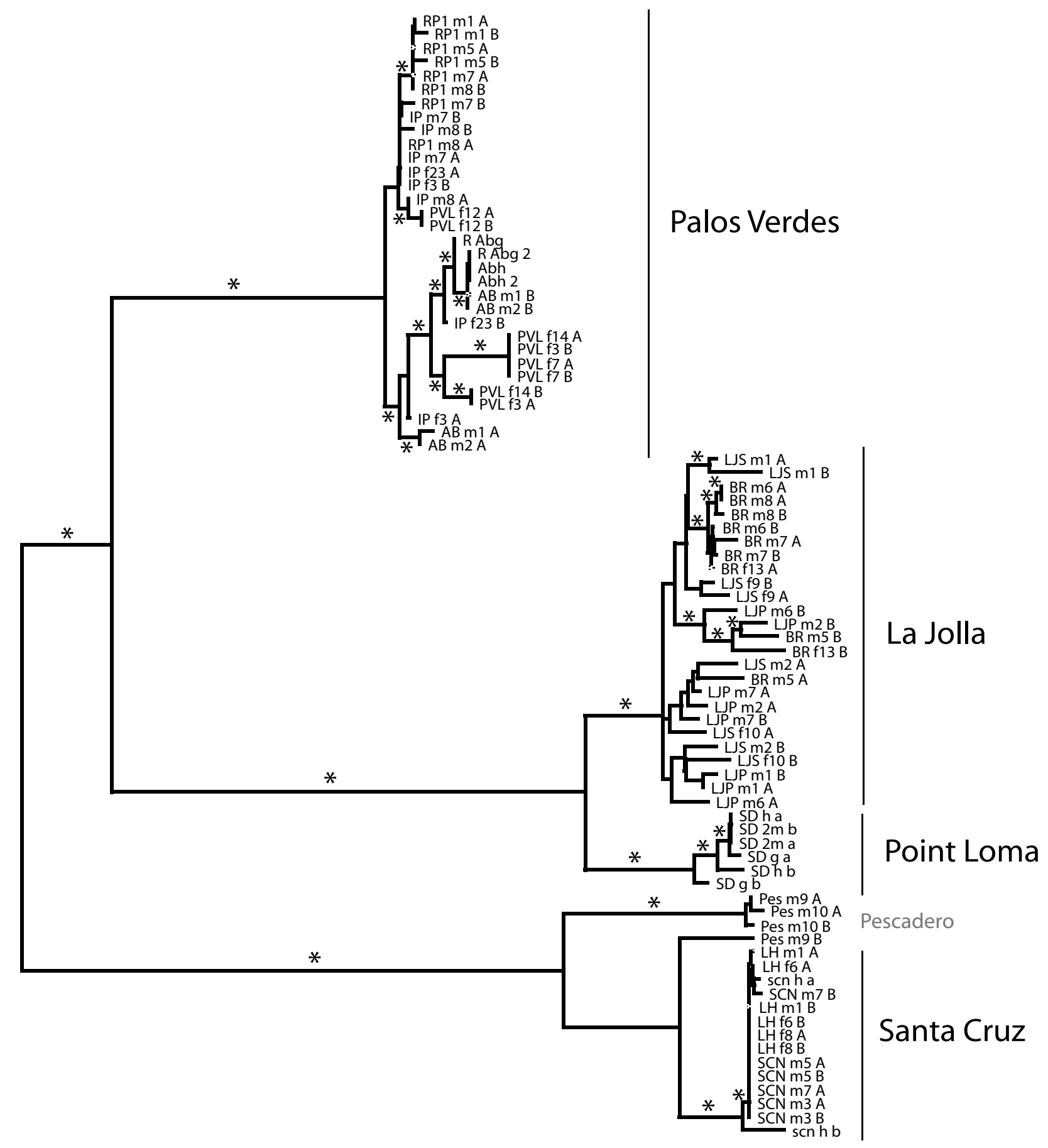

10 substitutions

Figure 6

NJ tree of RISP haplotypes from four focal regions for T. californicus. Tree is unrooted. Branches that were also supported by consensus trees of partial parsimony analyses of these RISP haplotypes are indicated by an *. We were unable to complete a parsimony analysis for the entire dataset but could complete analyses for pruned datasets to examine relationships between regions. Secondarily, the relationships of haplotypes within each region were separately analyzed using a parsimony method and compared to the relationships obtained from the $\mathrm{NJ}$ analysis. Intragenic recombination and reticulation of sequences within regions could make parsimony analyses difficult by yielding numerous equally parsimonious trees. 
Table 5: Polymorphism Within Regions and Sites for RISP.

\begin{tabular}{|c|c|c|c|c|c|c|c|c|c|c|c|c|c|c|}
\hline Outcrop & Site & Alleles & Hap. \# & Nonsyn' & Syn & Silent & Indel \# & $\pi_{\text {syn }}^{2}$ & $\pi_{\text {tot-sil }}$ & $\pi_{\text {total }}$ & Taj-D ${ }^{3}$ & $\mathrm{FW}-\mathrm{H}^{4}$ & $\mathrm{R}^{5}$ & $R_{m}{ }^{6}$ \\
\hline \multirow[t]{4}{*}{ Santa Cruz } & $\mathrm{SCN}$ & 8 & 3 & 0 & 2 & 5 & I & 0.00346 & 0.00093 & 0.00069 & $-1.60^{+}$ & -1.54 & 0 & 0 \\
\hline & $\mathrm{LH}$ & 6 & 2 & 0 & 0 & 0 & I & 0 & 0 & 0 & N/A & N/A & - & - \\
\hline & Total & 16 & 6 & 0 & 2 & 5 & 2 & 0.00198 & 0.00053 & 0.00040 & & & 0 & 0 \\
\hline & Avg. & & & & & & & 0.00173 & 0.00047 & 0.00035 & & & & \\
\hline \multirow{6}{*}{ Palos Verdes } & PVL & 8 & 3 & 0 & 2 & 15 & 2 & 0.00574 & 0.00639 & 0.00449 & 1.20 & -3.14 & 0.0028 & 0 \\
\hline & $A B I$ & 8 & 4 & 0 & 0 & 5 & 2 & 0 & 0.00189 & 0.00134 & 0.34 & -1.50 & 0.0004 & 0 \\
\hline & IP & 8 & 6 & 0 & 2 & 8 & 3 & 0.00348 & 0.00228 & 0.00162 & -0.86 & 1.71 & 0.0011 & 0 \\
\hline & RPI & 8 & 5 & 0 & I & 4 & I & 0.00294 & 0.00106 & 0.00075 & -1.03 & -0.21 & - & 0 \\
\hline & Total & 32 & 17 & 0 & 5 & 24 & 4 & 0.00745 & 0.00568 & 0.00404 & 0.15 & $-4.19^{+}$ & 0.0075 & 1 \\
\hline & Avg. & & & & & & & 0.00304 & 0.00291 & 0.00205 & & & & \\
\hline \multirow[t]{5}{*}{ La Jolla } & LJPI & 8 & 8 & 0 & I & 17 & 4 & 0.00170 & 0.00431 & 0.00315 & -0.89 & $-5.36 *$ & 0.0743 & 1 \\
\hline & LJS & 8 & 8 & 0 & I & 20 & 3 & 0.00167 & 0.00642 & 0.00462 & 0.07 & -0.29 & 0.1166 & 5 \\
\hline & $B R$ & 10 & 6 & 0 & 0 & 18 & 5 & 0 & 0.00430 & 0.00315 & -0.71 & $-5.24 *$ & 0.0009 & 5 \\
\hline & Total & 26 & 22 & 0 & 2 & 33 & 7 & 0.00367 & 0.00720 & 0.00425 & -0.68 & -2.46 & 0.0514 & 9 \\
\hline & Avg. & & & & & & & 0.00112 & 0.00501 & 0.00364 & & & & \\
\hline Point Loma & $\mathrm{SD}^{\circ}$ & 6 & 4 & 0 & 0 & 7 & 0 & 0 & 0.00236 & 0.00173 & -0.25 & $-4.53 *$ & - & - \\
\hline
\end{tabular}

IThe number of non-synonymous (nonsyn), synonymous (syn), and the combined non-coding and synonymous (silent).

${ }^{2}$ Average number of pairwise differences between sequences within a population for non-synonymous $\left(\pi_{\text {non-syn }}\right)$, synonymous $\left(\pi_{\text {syn }}\right)$, combined

synonymous and non-coding $\left(\pi_{\text {tot-sil }}\right)$, and all $\left(\pi_{\text {total }}\right)$ sites.

${ }^{3}$ Tajima's $D$ value, none were significant, but an ${ }^{+}$indicates $0.05<P<0.1$.

${ }^{4} \mathrm{Fay}$ and $\mathrm{Wu}$ 's $\mathrm{H}$ value with significance indicated by an $*(\mathrm{P}<0.05)$, and ${ }^{+}$indicating $0.05<\mathrm{P}<0.1$.

${ }^{5}$ Recombination parameter $(\mathrm{R})$ could not be estimated for some sites (-).

6 Minimum number of recombination events $\left(R_{m}\right)$ could not be estimated for some sites (-).

of Fay and Wu's H (with low levels of recombination used in coalescent simulations) are found for the BR (-5.24), LJP (-5.36), and SD (-4.53) sites (Table 4).

\section{Discussion}

Signals of population structure and history at a broad scale Although striking, the patterns of extreme genetic divergence observed among regional populations of T. californicus are not unprecedented, with high levels of mtDNA divergence found among populations in previous studies $[3,5,10,11]$. The high levels of genetic differentiation between $T$. californicus populations are likely to reflect generally low levels of gene flow between outcrops and their ability to persist on outcrops despite the ephemeral nature of individual copepod pools within a single outcrop during the course of a season $[14,15]$. In some cases genetic divergence is across short distances, for example there is 10 percent divergence in CYTB between the Point Loma and La Jolla regions despite the distance between these regions being equivalent to the distance between the end sites in the La Jolla region $(\sim 9 \mathrm{~km})$. At a slightly larger scale, the LagBch and Palos Verdes regions are 20 percent divergent and $53 \mathrm{~km}$ apart. Such patterns of high divergence in mtDNA genes have also been found in a congener, T. japon$i c u s$, with up to 28 percent divergence between populations in CYTB among locations in Japan and Korea [43].

Often the presence of highly genetically divergent lineages within a nominal species is thought to reflect the presence of cryptic species. Although defining cryptic species in allopatric populations is a difficult problem, a number of factors suggest that $T$. californicus may be still considered a single biological species (although they could potentially be considered separate species under other species definitions): These populations are able to interbreed in the lab and produce hybrids, including advanced generation hybrid lineages $[24,44]$, there is no evidence for premating isolation in crosses between populations $[45,46]$, and little consistent morphological divergence among populations has been found in studies done to date [12,47]. Other groups of copepods have also been found to have divergent genetic lineages that are often morphologically similar suggesting morphological stasis may be common in copepods [48-50]. In contrast to Tigriopus, in a number of other cases, genetically divergent lineages within a nominal species are sometimes found sympatrically distributed suggesting that these lineages do represent cryptic species [51-56]. Not all copepod species show these dramatic patterns of genetic differentiation between populations with some species having world-wide distributions and apparent genetic exchange between ocean basins [54,57].

Even though many comparisons of geographically distant populations of $T$. californicus show high levels of divergence, not all comparisons show this pattern, suggesting that occasionally gene flow can reach over longer spatial scales in this species. For example in the current dataset, LagBch and Point Loma are 2.3 percent divergent and 98 $\mathrm{km}$ apart, Dume and CAT are 3.0 percent divergent and 68 
km apart, and Santa Cruz and PES are 1.0 percent divergent and $47 \mathrm{~km}$ apart. In some cases these population comparisons are not the most geographically proximate, for example LagBch is much closer geographically to the other populations in the Palos Verdes region $(34 \mathrm{~km})$ rather than Point Loma populations $(107 \mathrm{~km})$. Edmands [5] found that from Oregon north to Alaska there was very little divergence in COI sequences among T. californicus populations and suggested that this could reflect recolonization after the last ice age. These seemingly contradictory patterns of low divergence in spite of extensive geographic separation between select populations of T. californicus within California may be indications of past long distance colonization events; thus, implying that although rare, long distance dispersal also plays a role in the evolutionary history of this species.

The degree of temporal stability of the outcrops and pools that comprise copepod habitat may play a large role in extinction/recolonization dynamics in $T$. californicus. Over short time scales, factors such as the number, size, and location of pools are likely to determine whether copepod habitat persists on an outcrop from year to year. At much longer timescales, sea level changes due to water storage in ice sheets during periods of glaciation will have major impacts on the distribution of available intertidal habitat. In fact, over the past 500000 years (500 kyr) sea levels have cycled repeatedly with sea levels in interglacial periods at or slightly above current levels and 100-150 m below current sea levels at glacial maxima. During the past $100 \mathrm{kyr}$ sea levels declined unevenly down to at least 100 $\mathrm{m}$ below the present sea level at the glacial maximum about 21 kyr [58] and then increased rapidly until about $8 \mathrm{kyr}$ when they reached their present levels (with potentially slightly higher levels in the mid-Holocene [59]). The distribution of available T. californicus habitat in the past would have been rather different from the present distribution with the current pool areas only inhabitable for at most 8 kyr. Recolonization of newly available outcrops would likely involve rare long-distance dispersal events from other outcrops. It is possible that some geographically disjunct areas with low levels of CYTB sequence divergence seen in the current dataset (e.g. Laguna Beach/ Point Loma and Catalina Island/Pt. Dume) could reflect the lack of available habitat in one region during the last glacial maximum followed by dispersal from other distant refugial populations once suitable habitat became available again.

In addition to the shifting availability of copepod habitat associated with sea level changes, the outcrops themselves are not stable over the longer time periods that would be required with standard mtDNA molecular clocks to produce the CYTB divergences uncovered among T. californicus populations. The uplifted headlands containing the rocky-intertidal regions in this study from Southern California do not appear to be more than one million years ( 1 myr) old. The Point Loma and La Jolla headlands resulted from uplift associated with the Rose Canyon fault leading to the emergence of these areas first as offshore islands less than one myr ago $[60,61]$. Similarly, the Palos Verdes region was at one point submerged and uplift resulted in its emergence as an island, also less than 1 myr ago, which was eventually connected to the mainland by the emergence of the Los Angeles basin [62]. Prior to that, it appears likely that both the San Diego and Los Angeles areas were extensively estuarine in nature until roughly 23 myr ago [63] and may not have supported much Tigriopus habitat. Interestingly, for several other coastal marine or intertidal animal taxa the Los Angeles region is the site of intra-specific phylogeographic breaks [64]. Given the relatively young age of the current rocky headlands in southern California, it is possible that the divergence in СYTB between copepods from nearly adjacent regions such as Point Loma and La Jolla ( 10 percent) or even Point Loma and Palos Verdes ( 20 percent) has accumulated over a period of less than $1 \mathrm{myr}$ if this divergence is not due to colonization from previously isolated source populations. With divergence times of 1 myr or less, these genetic divergences would reflect substantially elevated mutation rates for these copepod lineages (i.e. $>10$ percent/myr). The observation that mtDNA genes evolve much more rapidly than nuclear genes in T. californicus [10], could also support elevated absolute mtDNA rates in this taxon. However, given that long distance dispersal events seem to occur occasionally in this species, it is also possible that dispersal from genetically divergent source populations (perhaps populations now extinct) could explain the high levels of genetic divergence between these populations.

\section{Signals of population structure and history at a fine scale}

This study differs from previous datasets from T. californicus by determining the patterns of divergence in DNA sequence at a fine geographic scale (here for both a nuclear and a mtDNA gene). Although mtDNA sequence divergences in CYTB are much more modest within regions $(<1.3$ percent $)$, there is significant genetic structure between the majority of sites in all four regions as revealed by $\mathrm{F}_{\mathrm{ST}}$ distances and AMOVA (Table 2). The nested clade analyses support both historical separation and separation with limited gene flow between many clades within regions (Table 3); however, the reliability of individual inferences generated from nested clade analyses has been questioned [65]. We have done exploratory analyses using likelihood-based IM analyses on selected $T$. californicus sites under a model of isolation with gene flow using a MCMC method [66] and these analyses revealed both strong signals of limited gene flow between some sites and much higher gene flow between other sites con- 
sistent with results obtained from $\mathrm{F}_{\mathrm{ST}}$ and nested clade analyses (results not shown). Taken together, the $\mathrm{F}_{\mathrm{ST}}$ analyses, AMOVA, nested clade analyses, and limited IM analyses suggest significant historical separation and limited contemporary gene flow among a large fraction of these sites within each region.

An advantage of using CYTB over nuclear genes is that a mtDNA gene tree can yield insights into population history that may be lost due to recombination in nuclear gene trees. In each of the four regions the CYTB haplotype networks suggest slightly different patterns of historical isolation. The Palos Verdes region shows haplotype groupings that are largely geographically limited (Figure 5 ), the major exception to this is the FR2 haplotype that is closely related to the PVL haplotypes (and may be an example of fairly recent long-distance dispersal). These results suggest isolation resulting from geographic separation is dominant in shaping genetic diversity in this region and may reflect substantial isolation between these groups of sites since perhaps the last glacial maximum 21 kya or the return to present sea levels about 8 kya. The La Jolla CYTB haplotype network (Figure 4) suggests that there may have been significant historical isolation between at least three outcrop areas with only limited gene flow. For La Jolla, however, there are also several geographically separated sites (SIO, Naut, LJP3, and LJS) that show little evidence of isolation, a pattern that must reflect either on-going gene flow or recent colonization. Of the four sites, the LJS outcrop currently has the most extensive copepod habitat and could serve as a source for recolonization of the other sites after extinctions. Note that the SIO and LJS sites are not adjacent (LJP1/LJP2 sites are closer to SIO) suggesting that migration does not always occur in a stepwise fashion. For the Santa Cruz and Point Loma regions, although there is evidence for limited gene flow from $\mathrm{F}_{\mathrm{ST}}$ measures, CYTB haplotypes are either shared across many sites (Santa Cruz, Figure 3) or nested amongst one another (Point Loma, Figure 4). This pattern would seem most likely to reflect limited gene flow over short time scales but more mixing over longer periods, perhaps through occasional extinction and recolonization events of single outcrops.

There are substantial differences in the patterns of sequence divergence found between CYTB and RISP, with much greater levels of divergence among sites within a region for CYTB than for RISP. This lack of divergence is not due to a lack of variation in RISP, for the La Jolla and Palos Verdes regions, both average and total levels of polymorphism across sites within a region are similar for RISP and CYTB (Tables 1 and 4). In both of these regions phylogenetically distinct clusters of haplotypes from a limited number of sites are found for CYTB, while very few differences are fixed between sites within a region for
RISP. This difference between the two loci in amount of genetic differentiation suggests that the mtDNA-encoded CYTB is behaving as if it has a lower effective population size. If males and females have the same average reproductive success, then mtDNA would be expected to have roughly a fourth the effective population size of a nuclearencoded gene given its clonal, female-limited inheritance. In some cases the differences between the two markers are extreme (for example the LJP1/LJS comparison shows almost no structure at RISP, while LJP1 haplotypes form a distinct clade for CYTB, Figures 4 and 6). Geographicallylimited selective sweeps occurring anywhere on the linked mtDNA molecule are one possible explanation for a pattern of greater differentiation of mtDNA if the sweep causes polymorphic sites to become fixed within an outcrop for CYTB but does not affect RISP.

\section{Signals of non-neutral evolution}

Given the evidence outlined in the introduction for the accumulation of functional and fitness differences in mtDNA among populations of $T$. californicus it is instructive to look at the patterns of sequence variation to determine if signatures of non-neutral evolution can be found and if there is any evidence for coupled selection in both CYTB and RISP. For CYTB in the La Jolla and Santa Cruz regions, $\mathrm{MK}$ tests suggest an excess of replacement polymorphism, which could be attributable to segregating slightly deleterious mutations. The negative values for $\mathrm{Fu}$ and Li's D and F and Tajima's D tests indicate excess lowfrequency mutations in many of the sites as well, which could indicate some combination of a recovery from a recent selective sweep, expanding populations, or slightly deleterious mutations retained at a low frequency but unable to increase in frequency. Up to 20 of 29 non-synonymous polymorphisms within the three regions are found on terminal branches suggesting recent origin and many of these (10 of the 20) are singletons, indicating low frequency. It then appears that some fraction of the nonsynonymous polymorphism is likely to consist of slightly deleterious mutations that remain at low frequency and are generally quickly lost from populations. Polymorphism in mtDNA often shows an excess of slightly deleterious mutation in comparisons of polymorphism within and divergence within and between species [67]. These rare deleterious mutations are unlikely to impact inferences about population structure and historical relationships; however, they may impact the frequency spectrum of polymorphisms and produce excess low frequency variation that could otherwise suggest the hypothesis of a population expansion. Slightly deleterious mutations in mtDNA could also contribute to intergenomic coadaptation if they occasionally go to fixation (perhaps due to lowered effective population size during a population bottleneck) and then the fitness effects are subsequently compensated by changes in nuclear-encoded proteins of 
the electron transport system as suggested by the compensatory co-adaptation model [68].

Some of the non-synonymous polymorphisms within regions do not fit into the pattern of rare slightly deleterious mutation, for example, in the Santa Cruz region there are three non-synonymous substitutions of nine total substitutions occurring on the branches between the three most common haplotypes (Figure 2). A comparison of the divergence amongst these three haplotypes to the divergence to the Bodega Head outgroup in a variant of a $\mathrm{MK}$ test (6:3 vs. 149:6 synonymous: non-synonymous polymorphism vs. synonymous: non-synonymous fixed; $\chi^{2}$ test, $\mathrm{p}=0.03$ ) suggests that non-synonymous changes are in excess amongst the three Santa Cruz haplotypes. These polymorphisms are shared across sites at moderate frequencies which suggests that they are not deleterious mutations. Given the excess replacement variation seen on these internal branches compared to fixation to an outgroup, it is even possible that selection could be acting to maintain some non-synonymous variation at CYTB in this region.

Selective sweeps occurring on mtDNA can lower its apparent effective population size further and could contribute to faster fixation of unique haplotypes within species or populations. A few sites show significantly negative values for Fu and Li's F and D, Tajima's D tests, and significant Fay and $\mathrm{Wu} \mathrm{H}$ tests for CYTB that could indicate recent recovery from a selective sweep and could imply that selection has periodically driven advantageous mutations to near fixation (note that these mutations do not have to be in СYTB but could occur anywhere in the mtDNA). However, there are also demographic scenarios that could generate significant values for these tests; for example, significant Fay and Wu's $\mathrm{H}$ test values could potentially result from low levels of migration that introduce ancestral alleles back into a population at low frequencies. More extensive sampling of nuclear genes from these populations would be required to determine if a demographic scenario that affects all genes to some degree is likely to explain some of the deviations in allele frequencies found at CYTB for this handful of sites.

The RISP protein interacts structurally with CYTB in complex III and functionally as well through its iron-sulfur redox center, which is involved in the transfer of electrons in this complex [69]. Given this close association, evolution in the CYTB protein may be accompanied by coadaptation in RISP. The amino acid sequence of RISP is very well conserved across sites and regions in this dataset, in fact, the only polymorphic or fixed amino acid differences uncovered between sites and regions were the two amino acid differences between regions found previously for the sites $\mathrm{AB}, \mathrm{SCN}$, and SD by Willett and Burton [10]. Clearly a large amount of amino acid substitution can occur in CYTB with few changes in RISP amino acid sequence. Within regions we find amino acid changes in CYTB among some high frequency haplotypes ( 5 total in the Palos Verdes and Santa Cruz regions) and between certain regions (there are 6 amino acid differences in СYTB between La Jolla and Point Loma) with no changes in the RISP protein. These results indicate that structural co-evolution between these two proteins is not occurring within a region or between relatively closely related regions. These results do not rule out the possibility of CYTB coadaptation with other subunits of complex III (there are eight other nuclear-encoded subunits in this complex in most eukaryotes). At a broader scale, between the $\mathrm{AB}$ and SD sites, patterns of genetic interactions between RISP and two other complex III-associated genes (CYC and CYC1) suggest that there has been co-evolution between these three nuclear-encoded proteins, but the results also suggested that these interactions potentially did not involve CYTB for those two sites [27].

At the nucleotide sequence level for RISP there is some evidence for non-neutral patterns in allele frequencies in particular with the Fay and Wu's H test (for the BR, LJP, and SD sites). A potential explanation for this pattern is that these sites could have undergone a selective sweep in the region near RISP in the genome but demographic effects could provide an alternate explanation. One other unusual pattern for RISP is the extremely low level of polymorphism found in the SCN site (Table 4). This is unusual for this site as RISP has the lowest levels of silent/ non-coding polymorphism of the 11 genes examined to date for the SCN site $[10,70]$. In an HKA test [71] of polymorphism in these 11 genes at this site compared with divergence to the $\mathrm{AB}$ site, the RISP polymorphism is 4 -fold lower than expected and contributes to significantly heterogeneous patterns of variation at this site between these 11 genes (results not shown). This could be explained by a selective sweep having occurred near RISP in copepods from the Santa Cruz region removing variation in this gene. Overall, for both the RISP and CYTB genes the analyses of selection and deviations from neutrality indicate that although selection could occasionally impact patterns of variation at these genes in some populations, most of the phylogeographic patterns seen in this dataset are not likely to be the result of selection.

\section{Conclusion}

Given the dramatic patterns of genetic differentiation within and between regions for T. californicus, it is clear that gene flow between these sites is limited over short to moderate time spans. It appears likely that both the shorter-term processes of occasional extinction of all pools on single outcrops and subsequent recolonization, and the longer-term processes such as geological uplift 
and repeated cycles of sea level change with past episodes of glaciation have combined to shape patterns of genetic divergence in this species. If outcrop to outcrop dispersal is limited (as appears likely), the frequency with which all pools on an outcrop go extinct simultaneously on a single outcrop will determine the degree to which an outcrop can diverge from neighboring outcrops. Over longer periods of time, changes in sea levels during cycles of glaciation will radically alter the available intertidal habitat and limit the degree of divergence among outcrops within a region. Among regions the upper limit on the accumulation of genetic divergence appears to be generated by slower acting geological forces such as the uplift of coastline that has generated rocky intertidal areas in southern California over the last million years. However, even these lengths of divergence time between regions are unlikely to explain the high levels of divergence in $C Y T B$, and it is possible that there is an unusually high rate of mtDNA substitution in this species if these divergences are not due to colonization of these regions by previously isolated source populations.

A lack of gene flow among outcrops and potentially an elevated mtDNA substitution rate will not explain all of the patterns seen in the data; extinction events followed by long distance recolonization are also likely to play a significant role. The presence of shared haplotypes among multiple outcrops (not exclusively adjacent) is consistent with long-range colonization within regions. Geographically distant populations that show low levels of genetic divergence (some of which are more divergent from more proximate regions) suggest that past long-distance dispersal events occurred between these populations. These patterns set up a bit of a conundrum for this species with evidence for some long-distance dispersal suggesting the potential for gene flow but little gene flow over significant periods of time for comparisons of many nearby sites. One possibility is that long-distance dispersal is a very rare phenomenon and makes little contribution to genetically homogenizing disparate outcrops. It may also be possible that there is a priority effect such that the occasional migrant copepod rarely survives to reproduce upon reaching an inhabited outcrop but may survive and reproduce in uninhabited or sparsely inhabited pools.

Although selection does not appear to dramatically alter phylogeographic patterns at these genes, there are some signs from the data that it could be more subtly impacting the patterns of nucleotide variation in these copepod populations. Non-neutral patterns seen in this dataset are consistent with excess slightly deleterious polymorphism within regions and potential selective sweeps for a few sites. It is possible that these slightly deleterious polymorphisms could occasionally go to fixation during population bottlenecks and then contribute to a compensatory coevolution process between mtDNA and nuclearencoded proteins. Suggestions of selective sweeps from some sites come from frequency-based tests (Fu and Li's D and F, Tajima's D, and Fay and Wu's H), but for these tests it would be helpful to consider multiple genes to rule out potential demographic effects such as population expansion or contraction. Spatially-limited selective sweeps in mtDNA could potentially contribute to the greater divergence in CYTB than RISP between some sites. It is possible then that selection upon mtDNA could accentuate genetic divergence between select geographic sites or shift patterns of polymorphism within sites in this system, but it does not appear likely that this selection is radically altering the patterns of genetic variation within and between populations. Therefore, despite accumulating evidence of coadaptation between the nuclear- and mtDNA-encoded proteins of the electron transport system in this species, overall phylogeographic patterns in CYTB and RISP appear to be largely a product of history and not selection.

\section{Authors' contributions}

CSW conceived of the study, collected samples and sequence data, performed some analyses, and drafted manuscript. JTL collected sequence data, conducted analyses, and helped draft and revise the manuscript. Both authors read and approved the final manuscript.

\section{Additional material}

\section{Additional file 1}

Table S1. Sampling locations for Tigriopus californicus in southern and central California.

Click here for file

[http://www.biomedcentral.com/content/supplementary/1471-

2148-9-139-S1.pdf]

\section{Additional file 2}

Table S2. Primers used for PCR amplifications for the CYTB and RISP genes from $\mathrm{T}$. californicus.

Click here for file

[http://www.biomedcentral.com/content/supplementary/1471-

2148-9-139-S2.pdf]

\section{Additional file 3}

CYTB sequences in Arlequin format. This file contains the CYTB sequences from each of the four regions and additional sites (in non-interleaved Arlequin format).

Click here for file

[http://www.biomedcentral.com/content/supplementary/14712148-9-139-S3.doc]

\section{Additional file 4}

RISP sequences in Nexus format. This file contains the RISP sequences in nexus file format (interleaved sequences).

Click here for file

[http://www.biomedcentral.com/content/supplementary/14712148-9-139-S4.doc] 


\section{Additional file 5}

Table S3. Pairwise percent sequence divergence between selected haplotypes for CYTB.

Click here for file

[http://www.biomedcentral.com/content/supplementary/14712148-9-139-S5.pdf]

\section{Additional file 6}

Table S4. $F_{S T}$ values for CYTB from Arlequin based on pairwise comparisons of populations (pairwise sequence divergence).

Click here for file

[http://www.biomedcentral.com/content/supplementary/1471-

2148-9-139-S6.pdf]

\section{Additional file 7}

Table S5. Measures of the departures of the frequency spectra within regions and sites from neutral expectations.

Click here for file

[http://www.biomedcentral.com/content/supplementary/14712148-9-139-S7.pdf]

\section{Additional file 8}

Table S6. $F_{S T}$ values for RISP from Arlequin based on pairwise comparisons of populations (pairwise sequence divergence).

Click here for file

[http://www.biomedcentral.com/content/supplementary/14712148-9-139-S8.pdf]

\section{Additional file 9}

Table S7. RISP nucleotide and indel variation found in Santa Cruz region and PES for T. californicus.

Click here for file

[http://www.biomedcentral.com/content/supplementary/1471-

2148-9-139-S9.pdf]

\section{Additional file 10}

Table S8. RISP nucleotide and indel variation found in La Jolla region for T. californicus.

Click here for file

[http://www.biomedcentral.com/content/supplementary/14712148-9-139-S10.pdf]

\section{Additional file 11}

Table S9. RISP nucleotide and indel variation found in Palos Verdes region for $\mathrm{T}$. californicus.

Click here for file

[http://www.biomedcentral.com/content/supplementary/14712148-9-139-S11.pdf

\section{Acknowledgements}

We would like to thank M. Qureshi, N. Gindele, and M. Alexander for help with data collection. S. Edmands generously provided copepod samples from Catalina Island. Comments from several anonymous reviewers helped to improve the manuscript. Work supported by National Science Foundation grant DEB-0516139.

\section{References}

I. Burton RS: Differentiation and integration of the genome in populations of Tigriopus californicus. Evolution 1987, 41 :504-5I3.
2. Burton RS: Genetic evidence for long term persistence of marine invertebrate populations in an ephemeral environment. Evolution 1997, 51:993-998.

3. Burton RS: Intraspecific phylogeography across the Point Conception biogeographic boundary. Evolution 1998, 52:734-745.

4. Edmands S: Heterosis and outbreeding depression in interpopulation crosses spanning a wide range of divergence. Evolution 1999, 53:1757-1768.

5. Edmands S: Phylogeography of the intertidal copepod Tigriopus californicus reveals substantially reduced population differentiation at northern latitudes. Mol Ecol 2001, 10:1743-1750.

6. Burton RS, Ellison CK, Harrison JS: The sorry state of F2 hybrids: Consequences of rapid mitochondrial DNA evolution in allopatric populations. Am Nat 2006, 168:SI4-S24.

7. Willett CS: No evidence for faster male hybrid sterility in population crosses of an intertidal copepod (Tigriopus californicus). Genetica 2008, I33:129-136.

8. Burton RS, Feldman MW, Curtsinger JW: Population genetics of Tigriopus californicus. Copepoda: Harpacticoida.: I. Population structure along the central California coast. Mar Ecol Prog Series 1979, I:29-39.

9. Burton RS, Swisher SG: Population structure of the intertidal copepod Tigriopus californicus as revealed by field manipulation of allele frequencies. Oecologia 1984, 65:108-III.

10. Willett CS, Burton RS: Evolution of interacting proteins in the mitochondrial electron transport system in a marine copepod. Mol Biol Evol 2004, 21 :443-453.

II. Rawson PD, Burton RS: Molecular evolution at the cytochrome c oxidase subunit 2 gene among divergent populations of the intertidal copepod, Tigriopus californicus. I Mol Evol 2006, 62:753-764.

12. Monk CR: Marine Harpacticoid Copepods from California. Trans Amer Microscop Soc 1941, 60:75-99.

13. Burton RS, Feldman MW: Population genetics of Tigriopus californicus. II. Differentiation among neighboring populations. Evolution 1981, 35: I | 92-I 205.

14. Dybdahl MF: Extinction, recolonization, and the genetic structure of tidepool copepod popululations. Evolutionary Ecology 1994, 8: I I3-124.

15. Powlick Jj: Seasonal abundance and population flux of Tigriopus californicus (Copepoda: Harpacticoida) in Barkley sound, British Columbia. J Mar Biol Assoc UK 1998, 78:467-48I.

16. Nabholz B, Glémin S, Galtier N: Strong variations of mitochondrial mutation rate across mammals - the longevity hypothesis. Mol Biol Evol 2008, 25: I 20-130.

17. Lynch M, Koskella B, Schaak S: Mutation pressure and the evolution of organelle genomic architecture. Science 3I I: I727-I730.

18. Haag-Liautard C, Coffey N, Houle D, Lynch M, Charlesworth B, Keightley PD: Direct estimation of the mitochondrial DNA mutation rate in Drosophila melanogaster. PLoS Biology 2008, 6:1706-17|4

19. Edmands S, Burton RS: Cytochrome c oxidase activity in interpopulation hybrids of a marine copepod: A test for nuclearnuclear or nuclear-cytoplasmic coadaptation. Evolution 1999 , 53:1972-1978.

20. Rawson PD, Burton RS: Functional coadaptation between cytochrome $\mathrm{c}$ and cytochrome $\mathrm{c}$ oxidase within allopatric populations of a marine copepod. Proc Natl Acad Sci USA 2002, 99: 12955-12958.

21. Burton RS, Ellison CK: Disruption of mitochondrial function in interpopulation hybrids of Tigriopus californicus. Evolution 2006, 60:1382-1391.

22. Harrison JS, Burton RS: Tracing hybrid incompatibilities to single amino acid substitutions. Mol Biol Evol 2006, 23:559-564.

23. Willett CS, Burton RS: Viability of cytochrome $\mathbf{c}$ depends on cytoplasmic background in Tigriopus californicus. Evolution 200I, 55:1592-I599.

24. Willett CS, Burton RS: Environmental influences on epistatic interactions: Viabilities of cytochrome c genotypes in interpopulation crosses. Evolution 2003, 57:2286-2292.

25. Burton RS, Ellison CK: Genotype-dependent variation of mitochondrial transcriptional profiles in interpopulation hybrids. Proc Natl Acad Sci 2008, I 05: 1583 I- 15836.

26. Burton RS, Ellison CK: Interpopulation hybrid breakdown maps to the mitochondrial genome. Evolution 2008, 62:63I-638. 
27. Willett CS: Deleterious epistatic interactions between electron transport system protein-coding loci in the copepod Tigriopus californicus. Genetics 2006, I 73:|465-|477.

28. Willett CS: Significant variation for fitness impacts of ETS loci in hybrids between populations of Tigriopus californicus. Journal of Heredity 2008, 99:56-65.

29. Rozas J, Rozas R: DnaSP version 3: an integrated program for molecular population genetics and molecular evolution analysis. Bioinformatics 1999, I 5: |74- I75.

30. Tajima F: Statistical methods for testing the neutral mutation hypothesis by DNA polymorphism. Genetics 1989, I 23:585-595

31. Fu YX, Li W-H: Statistical tests of neutrality of mutations. Genetics 1993, I33:693-709.

32. Hudson RR: Estimating the recombination parameter of a finite population model without selection. Genet Res 1987 50:245-250.

33. Hudson RR, Kaplan NL: Statistical properties of the number of recombination events in the history of a sample of DNA sequences. Genetics 1985, I I I: 147-164.

34. Fay JC, Wu C-I: Hitchhiking under positive Darwinian selection. Genetics 2000, 155:1405-1413.

35. McDonald JH, Kreitman M: Adaptive protein evolution at the Adh locus in Drosophila. Nature I991, 35 I:652-654.

36. Excoffier L, Laval G, Schneider S: Arlequin ver. 3.0: An integrated software package for population genetics data analysis. Evolutionary Bioinformatics Online 2005, I:47-50.

37. Jensen JL, Bohonak AJ, Kelley ST: Isolation by distance, web service. BMC Genetics 2005, 6: 13 [http://ibdws.sdsu.edu/].

38. Posada D, Crandall KA, Templeton AR: GeoDis: A program for the Cladistic Nested Analysis of the Geographical Distribution of Genetic Haplotypes. Mol Ecol 2000, 9:487-488.

39. Templeton AR: Nested clade analyses of phylogeographic data: testing hypotheses about gene flow and population history. Mol Ecol 1998, 7:38|-397.

40. Clement $M$, Posada D, Crandall KA: TCS: a computer program to estimate gene genealogies. Mol Ecol 2000, 9: I657-1660.

41. Swofford DL: PAUP*: Phylogenetic Analysis Using Parsimony *and Other Methods. Sinauer Associates, Sunderland, MA., Version $4 ; 2001$.

42. Ronquist F, Huelsenbeck JP: MRBAYES 3: Bayesian phylogenetic inference under mixed models. Bioinformatics 2003, 19:1572-1574

43. Jung SO, Lee YM, Park TJ, Park HG, Hagiwara A, Leung KMY, Dahms $\mathrm{H}-\mathrm{U}$, Lee $\mathrm{W}$, Lee J-S: The complete mitochondrial genome of the intertidal copepod Tigriopus sp.. Copepoda, Harpactidae. from Korea and phylogenetic considerations. J Exp Mar Biol Ecol 2006, 333:25I-262.

44. Edmands S, Feaman HV, Harrison JS, Timmerman CC: Genetic con sequences of many generations of hybridization between divergent copepod populations. J Heredity 2004, 96: I |4- I 23.

45. Ganz HH, Burton RS: Genetic differentiation and reproductive incompatibility among Baja California populations of the copepod Tigriopus californicus. Mar Biol 1995, I 23:821-828.

46. Palmer CA, Edmands S: Mate choice in the face of both inbreeding and outbreeding depression in the intertidal copepod Tigriopus californicus. Mar Biol 2000, I36:693-698.

47. Edmands S, Harrison JS: Molecular and quantitative trait variation within and among populations of the intertidal copepod Tigriopus californicus. Evolution 2003, 57:2277-2285.

48. Dodson SI, Grishanin AK, Gross K, Wyngaard GA: Morphological analysis of some cryptic species in the Acanthocyclops vernalis species complex from North America. Hydrobiologia 2003, 500: $|3|-\mid 43$.

49. Lee CE, Frost BW: Morphological stasis in the Eurytemora affinis species complex (Copepoda: Temoridae). Hydrobiologia 2002, 480: III-I28.

50. Thum RA, Harrison RG: Deep genetic divergences among mor phologically similar and parapatric Skistodiaptomus (Copepoda: Calanoida: Diaptomidae) challenge the hypothesis of Pleistocene speciation. Biological J Linnean Soc 2009, 96: I50-165.

51. Lee CE: Global phylogeography of a cryptic copepod species complex and reproductive isolation between genetically proximate "populations". Evolution 2000, 54:2014-2027.

52. Rocha-Olivares A, Fleeger JW, Foltz DW: Decoupling of molecular and morphological evolution in deep lineages of a meiobenthic harpacticoid copepod. Mol Biol Evol 200I, 18:1088-1102.

53. Schizas NV, Coull BC, Chandler GT, Quattro JM: Sympatry of distinct mitochondrial DNA lineages in a copepod inhabiting estuarine creeks in the southeastern USA. Marine Biology 2002, | 40:585-594.

54. Goetze E: Cryptic speciation on the high seas; global phylogenetics of the copepod family Eucalanidae. Proc $R$ Soc B 2003, 270:232|-233|.

55. Caudill CC, Bucklin A: Molecular phylogeography and evolutionary history of the estuarine copepod, Acartia tonsa, on the Northwest Atlantic coast. Hydrobiologia 2004, 5 I I:91-102.

56. Chen G, Hare MP: Cryptic ecological diversification of a planktonic estuarine copepod, Acartia tonsa. Molecular Ecology 2008 | 7:|45|-|468.

57. Eberl R, Cohen S, Cipriano F, Carpenter EJ: Genetic diversity of the pelagic harpacticoid copepod Macrosetella gracilis on colonies of the cyanobacterium Trichodesmium spp. Aquatic Biology 2007, I:33-47

58. Rabineau M, Berné S, Olivet JL, Aslanian D, Guillocheau F, Joseph P. Paleo sea levels reconsidered, direct observation of paleoshoreline position during glacial maxima for the last $\mathbf{5 0 0 , 0 0 0}$ yr. Earth Planet Sci Let 2006, 252: I I9-I 37

59. Peltier WR: On eustatic sea level history: Last glacial maximum to Holocene. Quaternary Sci Rev 2002, 2 I:377-396.

60. Abbott P: The Rose canyon fault. Environment Southwest 1989 , 524:I-7.

6I. Kern JP, Rockwell TK: Chronology and deformation of quaternary marine shorelines, San Diego County, California. In Quaternary coasts of the United States: Marine and lacustrine systems Edited by: Fletcher CH, Wehmiller JF. Tulsa: SEPM; 1992:377-382

62. Ward SN, Valensise G: The Palos Verdes terraces, California: bathtub rings from a buried reverse fault. J Geophys Res 1994, 99:4485-4494.

63. Jacobs DK, Haney TA, Louie KD: Genes, diversity, and geologic process on the Pacific coast. Ann Rev Earth Planet Sci 2004 32:601-652.

64. Dawson MN: Phylogeography in coastal marine animals: a solution from California? J Biogeography 200I:723-736.

65. Panchal M, Beaumont MA: The automation and evaluation of nested clade phylogeographic analysis. Evolution 2007, 6l:|466-1480.

66. Hey J, Nielsen R: Multilocus methods for estimating population sizes, migration rates and divergence time, with applications to the divergence of Drosophila pseudoobscura and D. persimilis. Genetics 2004, 167:747-760.

67. Rand DM, Kann LM: Excess amino acid polymorphism in mitochondrial DNA: Contrasts among genes from Drosophila, mice, and human. Mol Biol Evol 1996, I3:735-748

68. Rand DM, Haney RA, Fry A): Cytonuclear coevolution: the genomics of cooperation. Trends Ecol Evol 2004, 19:645-653.

69. Saraste M: Oxidative phosphorylation at the fin de siecle. Science 1999, 283:|488-|493.

70. Willett CS, Berkowitz JN: Viability effects and not meiotic drive cause dramatic departures from Mendelian inheritance for malic enzyme in hybrids of Tigriopus californicus populations. J Evol Biol 2007, 20: I | 96-I205.

7I. Hudson RR, Kreitman M, Aguadé M: A test of neutral molecular evolution based on nucleotide data. Genetics 1987. I 16:153-159. 